\title{
Transient Quantum Beat Oscillations in Extreme-Relativistic Diffraction in Time
}

\author{
Salvador Godoy \\ Universidad Nacional Autónoma de México, Facultad de Ciencias, Física, CDMX, Coyoacán, México \\ Email: sgs@ciencias.unam.mx
}

How to cite this paper: Godoy, S. (2021) Transient Quantum Beat Oscillations in Extreme-Relativistic Diffraction in Time. Journal of Modern Physics, 12, 1-9. https://doi.org/10.4236/jmp.2021.121001

Received: November 24, 2020

Accepted: January 3, 2021

Published: January 6, 2021

Copyright $\odot 2021$ by author(s) and Scientific Research Publishing Inc. This work is licensed under the Creative Commons Attribution International License (CC BY 4.0). http://creativecommons.org/licenses/by/4.0/

\begin{abstract}
In the solution of the Klein-Gordon equation for the shutter problem, we prove that, at internuclear distances, a relativistic beam of Pi-mesons has a probability density which oscillates in time in a similar way to the spatial dependence in optical Fresnel diffraction from a straight edge. However, for an extreme-relativistic beam, the Fresnel oscillations turn into quantum damped beat oscillations. We prove that quantum beat oscillations are the consequence, at extreme-relativistic velocities, of the interference between the initial incident wave function, and the Green's function in the relativistic shutter problem. This is a pure quantum relativistic phenomenon.
\end{abstract}

\section{Keywords}

Quantum Beat Oscillations, Relativistic Diffraction in Time

\section{Introduction}

Quantum beat oscillations are a common subject in Atomic and Molecular Spectroscopy. In Atomic physics, the term quantum beat refers to a superposed oscillatory behavior in the light intensity emitted by some suddenly excited atomic systems in their subsequent decay [1]. In Molecular Spectroscopy, quantum beat spectroscopy is a Doppler-free time domain method based on the creation of molecular coherences with a laser pulse and the measurement of their subsequent time evolution [2].

In this same context is the work of Villavicencio et al. [3], where transient phenomena of phase-modulated cutoff wave packets were explored by deriving an exact general solution to Schrödinger's equation for finite-range potentials involving arbitrary initial quantum states. They show that the dynamical features of the probability density are governed by a virtual two-level system. They also 
found that for a system with a bound state the interplay between the virtual levels with the latter causes a quantum beat. They also find a regime characterized by a time-diffraction oscillation. This last result came as a big surprise; it happens that in the exact Schrödinger's solution of the shutter problem [4], where we find diffraction in time as a consequence of the free time-evolution of an initial space-discontinuous beam of particles, the exact analytic expression for the probability density has a structure in which quantum beats do not exist.

Diffraction in time oscillations is a pure quantum phenomenon, and similar oscillations arise at the moment of closing and opening gates in nanoscopic circuits [5]. With adequate potentials added to the model, it has been used to study transient dynamics of tunneling matter waves [6], and the transient response to abrupt changes of the interaction potential in semiconductor structures and quantum dots [7]. For a review on the subject see [8] [9]. There is, in summary, a strong motivation for a thorough understanding of transient time oscillation of beams of matter.

In this paper we address the following question: are there quantum shutter solutions (not in the Schrödinger's equation) where quantum beats substitute the usual Fresnel oscillations of diffraction in time? In this paper, we report the positive finding of such result in the diffraction in time of an extreme-relativistic beam of free particles.

The main contribution of this paper is to show the existence of transient quantum beats in the extreme-relativistic quantum shutter problem. After solving the Klein-Gordon equation for the 1D shutter problem, we prove that, at internuclear distances, a relativistic beam of neutral Pi-mesons, has a probability density which oscillates in time in a similar way to the Fresnel oscillations derived in the Schrödinger equation. However, for an extreme-relativistic beam, the Fresnel oscillations turn into quantum damped beats!

\section{The Klein-Gordon Shutter Problem}

In the relativistic shutter problem for spin-0 particles, we assume for all $t \leq 0$, that we have a discontinuous right-moving plane wave in the left side of a perfectly absorbing shutter, and zero to the right:

$$
\psi(x, t \leq 0)=\left\{\begin{array}{ll}
\mathrm{e}^{i(k x-\omega t)} & x \leq 0 \\
0 & x>0
\end{array}=\mathrm{e}^{i(k x-\omega t)} \theta(-x),\right.
$$

here $\theta(x)$ denotes the step function, and the angular frequency is given by $\omega=c\left(k^{2}+\mu^{2}\right)^{1 / 2}$ and $\mu \equiv m c / \hbar$. At $t=0$, when the shutter is suddenly opened, we have the initial conditions:

$$
\psi(x, 0)=\mathrm{e}^{i k x} \theta(-x), \quad \frac{\partial \psi(x, 0)}{\partial t}=-i \omega \mathrm{e}^{i k x} \theta(-x),
$$

which we use to solve the Klein-Gordon (K-G) equation,

$$
\frac{\partial^{2} \psi}{\partial x^{2}}-\frac{\partial^{2} \psi}{\partial(c t)^{2}}=\mu^{2} \psi
$$


The exact solution of this initial-value problem is given in the appendix A, where we use dimensionless variables for "position" $\chi$ and "time" $\tau$ defined by

$$
\chi \equiv \mu x=\frac{m c}{\hbar} x, \quad \tau \equiv \mu c t=\frac{m c^{2}}{\hbar} t .
$$

For arbitrary $(\tau>0, \quad \chi>0)$, on the right-hand side of the shutter, we have the exact $\mathrm{K}-\mathrm{G}$ wave function solution:

$$
\begin{aligned}
\frac{2 \mathrm{e}^{i \Omega \tau}}{\theta(\tau-\chi)} \psi(\chi, \tau ; \Omega) & =\mathrm{e}^{i \Omega \chi}-\int_{\chi}^{\tau} \mathrm{d} u \mathrm{e}^{i \Omega u}\left\{\chi \frac{J_{1}[\xi(\chi, u)]}{\xi(\chi, u)}+i \kappa J_{0}[\xi(\chi, u)]\right\} \\
& \equiv \mathrm{e}^{i \Omega \chi}+i \kappa\left(C_{0}+i S_{0}\right)-\chi\left(C_{1}+i S_{1}\right)
\end{aligned}
$$

Here, we have defined both dimensionless "wave-number" $\kappa$ and "angular-frequency" $\Omega$ as:

$$
\xi(\chi, \tau) \equiv \sqrt{\tau^{2}-\chi^{2}}, \quad \kappa \equiv \frac{k}{\mu}=\frac{v / c}{\sqrt{1-(v / c)^{2}}}, \quad \Omega \equiv \frac{\omega}{\mu c}=\frac{1}{\sqrt{1-(v / c)^{2}}} .
$$

We have also defined, the real functions: $\left(C_{0}, S_{0}, C_{1}, S_{1}\right)$ given by:

$$
\begin{gathered}
C_{0}(\chi, \tau ; \Omega)+i S_{0}(\chi, \tau ; \Omega) \equiv \theta(\tau-\chi) \int_{\chi}^{\tau} \mathrm{e}^{i \Omega u} J_{0}[\xi(\chi, u)] \mathrm{d} u, \\
C_{1}(\chi, \tau ; \Omega)+i S_{1}(\chi, \tau ; \Omega) \equiv \theta(\tau-\chi) \int_{\chi}^{\tau} \mathrm{e}^{i \Omega u} \frac{J_{1}[\xi(\chi, u)]}{\xi(\chi, u)} \mathrm{d} u .
\end{gathered}
$$

\section{Time-Dependent Density}

Given the K-G wave function $\psi(x, t)$, the probability density is calculated by:

$$
\rho(x, t)=\frac{i \hbar}{2 m c^{2}} \psi^{*} \frac{\partial \psi}{\partial t}+c . c .,
$$

which, for dimensionless variables, turn into $\rho(\chi, \tau)$, we have:

$$
\rho(\chi, \tau)=\frac{i}{2} \psi^{*}(\chi, \tau) \frac{\partial \psi(\chi, \tau)}{\partial \tau}+\text { c.c. }
$$

Notice that the initial relativistic plane-wave, $\psi(\chi, \tau \leq 0)=\exp [i(\kappa \chi-\Omega \tau)]$, does not have unit density, in fact we have

$$
\rho(\chi, \tau \leq 0)=\Omega \text {. }
$$

This means, as we will show, that the time-evolved density $\rho(\chi>0, \tau>0)$ will oscillate in time around the initial value: $\rho=\Omega$. Using Equation (5) we get for arbitrary $(\tau>0, \quad \chi>0)$ the exact K-G probability density:

$$
\begin{aligned}
& \frac{8 \rho(\chi, \tau)}{\Omega \theta(\tau-\chi)} \\
& =\left[\mathrm{e}^{i \Omega \chi}-\chi\left(C_{1}+i S_{1}\right)-i \kappa\left(C_{0}+i S_{0}\right)-i \frac{\chi}{\Omega} \frac{J_{1}(\xi)}{\xi} \mathrm{e}^{i \Omega \tau}+\frac{\kappa}{\Omega} J_{0}(\xi) \mathrm{e}^{i \Omega \tau}\right] \\
& \quad \times\left[\mathrm{e}^{-i \Omega \chi}-\chi\left(C_{1}-i S_{1}\right)+i \kappa\left(C_{0}-i S_{0}\right)\right]+c . c .
\end{aligned}
$$


The presence of the function $\theta(\tau-\chi)$ implies, as expected, that for times $0 \leq t \leq x / c$ the density vanishes, in full agreement with the relativity theory.

\section{Relativistic Diffraction in Time for Pi-Mesons}

At this point, we apply our present results to a relativistic beam of neutral Pi-mesons $\left(\pi^{0}\right)$ which are 0 -spin particles. We know that Pi-mesons are the carriers of nuclear forces and for this, and no other reason, we assume a particle detector fixed at a distance $x$ from the shutter of about, $x=1.7 \times 10^{-15} \mathrm{~m}$, where nuclear forces have a maximum range. All mesons are unstable and decay in various ways. Neutral Pi-mesons have a mean-life given by: $t^{0} \approx 10^{-17} \mathrm{sec}$, which for a mass of, $m=135 \mathrm{MeV} / \mathrm{c}^{2}$, we have, $\tau^{0} \approx 10^{+7}$. Consequently, our theoretical predictions for diffraction in time for neutral Pi-mesons will make sense only if the predicted transient behavior happens in a range of times $\Delta \tau$ having orders of magnitude less than $\tau^{0}$. Next, we will show that for all assumed conditions of position and energy, this condition is well satisfied.

Let us assume a relativistic beam of monochromatic, $\omega=c\left(k^{2}+\mu^{2}\right)^{1 / 2}$, $\mu \equiv m c / \hbar$, neutral Pi-mesons ( $m=135 \mathrm{MeV} / \mathrm{c}^{2}$ ) with "low" velocities, $v / c=0.5$, and a particle detector at, $x=1.7 \times 10^{-15} \mathrm{~m}$. Using the exact result in Equation (12) we show in Figure 1 the plot of the relativistic probability density versus time.

The main features of this plot are: 1) As expected, for times $0 \leq t \leq x / c$ the density vanishes. 2) In spite of the fact that the exact relativistic density given in Equation (12) looks different from the Schrödinger one, however, for $t>x / c$ the damped oscillations we see in Figure 1 look similar to the ones predicted in the optical Fresnel diffraction by a straight edge [10]. We claim that diffraction in time is present in relativistic quantum mechanics! 3) We measure the period of one initial oscillation, $\Delta \tau=41$, which corresponds to $\Delta t=2 \times 10^{-22} \mathrm{sec}$ ! This range is well below the $\pi^{0}$-meson's mean-life: $t^{0} \approx 10^{-17} \mathrm{sec}$.

\section{Damped Beats in Extreme-Relativistic Diffraction in Time}

The next obvious question is: how does diffraction in time look like for extreme-

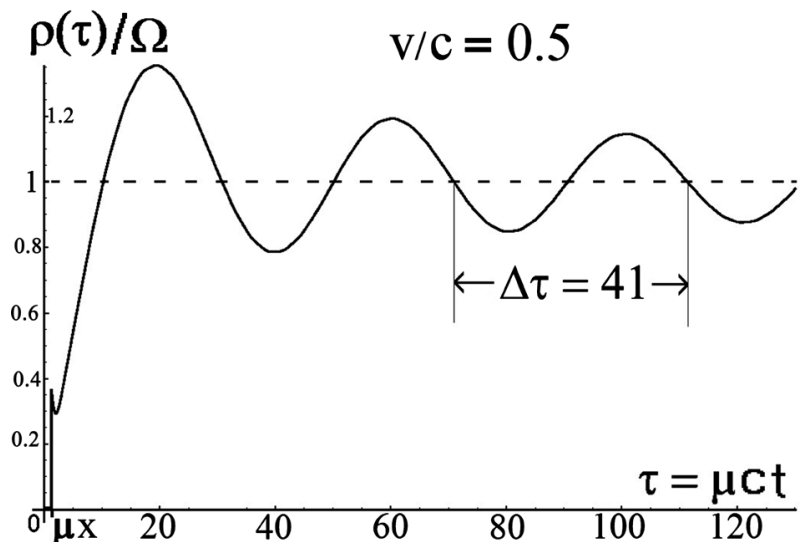

Figure 1. Relativistic diffraction in time of Pi-mesons, $v / c=0.5$. 
relativistic values of $v / c$ ? In this section, we show properties of extreme relativistic diffraction in time which, as far as we know, have never been reported before.

As we gradually increase the relativistic velocities $v / c$, the diffraction in time pattern gradually changes from Fresnel into damped beats! To illustrate this, in Figure 2, we have chosen the particular case of an extreme-relativistic beam of neutral Pi-mesons, $v / c=0.99$. Assuming the particle detector at a fixed distance, $x=1.7 \times 10^{-15} \mathrm{~m}$, we plot as a function of time the K-G shutter density $\rho(\chi, \tau)$, given in Equation (12). We clearly see the gradual distorsion of the Fresnel oscillatios.

Finally in Figure 3 for a more energetic beam, $v / c=0.998$, we clearly see the total transformation of the Fresnel oscillations into a perfect case of quantum beats. Here for the slow, modulating amplitude, we measure its period:

$\Delta \tau_{\text {slow }}=6$, which means a "slow" oscillation with a modulating period, $T_{\text {slow }}=3 \times 10^{-23} \mathrm{sec}$ and an angular frequency $\omega_{\text {slow }}=2 \times 10^{+23} \mathrm{sec}^{-1}$. As for the fast oscillations we have $\Delta \tau_{\text {fast }}=0.4$, implying a shorter period,

$T_{\text {fast }}=2 \times 10^{-24} \mathrm{sec}$ and a faster angular frequency: $\omega_{\text {fast }}=3 \times 10^{+24} \mathrm{sec}^{-1}$. All periods are shorter than the $\pi^{0}$-meson's mean-life: $t^{0} \approx 10^{-17} \mathrm{sec}$.

\section{Conclusions}

Two simultaneous properties are needed for the existence of quantum beats. The

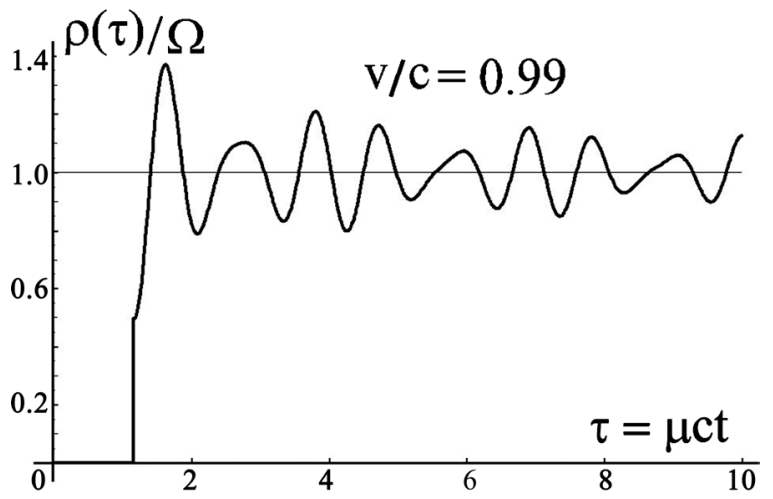

Figure 2. Deformed Fresnel oscillations for extreme relativistic Pi-mesons.

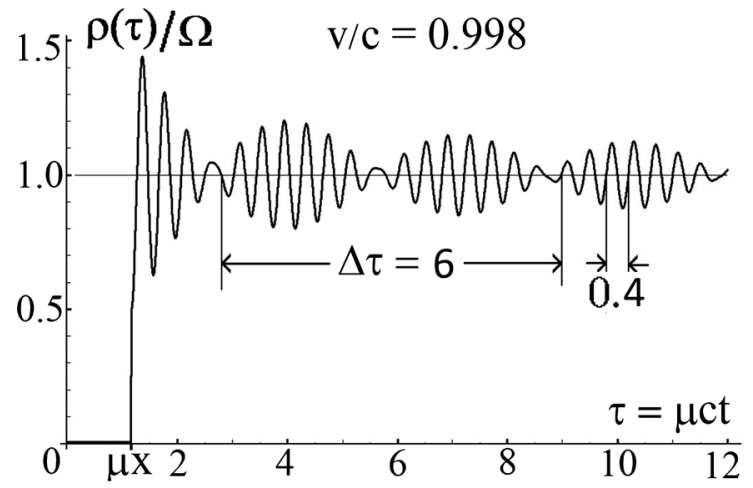

Figure 3. Transient quantum beats for extreme relativistic Pi-mesons. 


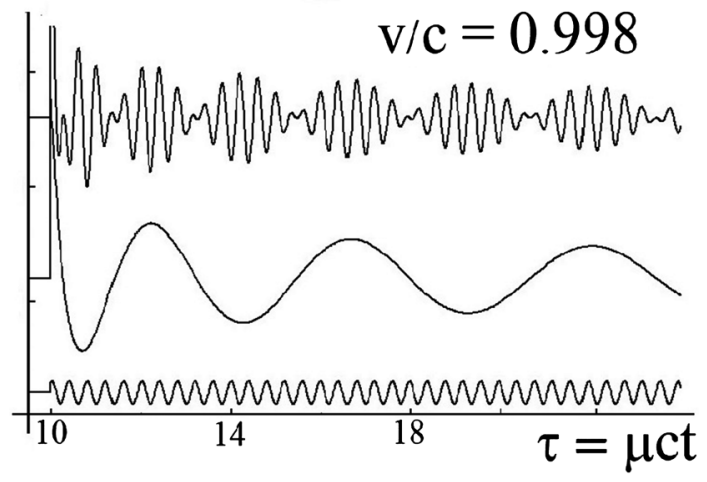

Figure 4. From bottom to top: the trigonometric, the Bessel, and the product.

first is that in the probability density, which is a quadratic expression of the wave function, we have products of two different oscillatory functions. In our case in Equation (12) we have the most important contribution of these products:

$$
[\sin (\Omega \tau)+\cos (\Omega \tau)] J_{0}\left(\sqrt{\tau^{2}-\chi^{2}}\right) \quad(\tau \geq \chi)
$$

where the agular frequency $\Omega$ is given by $\Omega \equiv \frac{1}{\sqrt{1-(v / c)^{2}}}$.

The second property is that only for extreme relativistic velocities the two oscillatory functions must have similar angular frequencies. Here, for $v / c=0.998$, ( $\Omega=15$ ), the Bessel $J_{0}$ becomes a slow oscillating function of time and each trigonometric is a fast one. See Figure 4. The products show damped beats! However, for $v / c<0.9$, the beats are no longer present.

In conclusion, quantum beat oscillations are the consequence, at extreme relativistic velocities, of the interference between the initial incident wave function: $\mathrm{e}^{i \Omega \tau}$, and the Green's function of the relativistic shutter problem: $J_{0}\left(\tau^{2}-\chi^{2}\right)$. This a pure quantum relativistic phenomenon. We claim that our result is original and has never been reported before.

\section{A. The Klein-Gordon Solution for the 1D Shutter Problem}

Let us consider the Klein-Gordon (K-G) equation for $\psi(\chi, \tau)$ defined in the infinite range $-\infty<\chi<\infty$,

$$
\frac{\partial^{2} \psi}{\partial \chi^{2}}-\frac{\partial^{2} \psi}{\partial \tau^{2}}=\psi
$$

We demand bounded boundary conditions: $\lim _{|x| \rightarrow \infty}|\psi(\chi, \tau)|<\infty$, and the initial conditions, corresponding to the shutter problem:

$$
\psi(\chi, 0)=\mathrm{e}^{i \kappa \chi} \theta(-\chi), \quad \frac{\partial \psi(\chi, 0)}{\partial \tau}=-i \Omega \mathrm{e}^{i \kappa \chi} \theta(-\chi)
$$

where $\Omega \equiv \sqrt{1+\kappa^{2}}$.

To solve the K-G equation, we begin taking the Laplace transform of Equation (14). Denoting, 


$$
\phi(\chi, s) \equiv \mathcal{L}[\psi(\chi, \tau)] \equiv \int_{0}^{\infty} \mathrm{e}^{-s \tau} \psi(\chi, \tau) \mathrm{d} \tau,
$$

we find the differential equation

$$
\frac{\mathrm{d}^{2} \phi(\chi, s)}{\mathrm{d} \chi^{2}}-\left(s^{2}+1\right) \phi(\chi, s)=-(s-i \Omega) \mathrm{e}^{i \kappa \chi} \theta(-\chi)
$$

which holds in the range $-\infty<\chi<\infty$.

Due to the presence of the step function $\theta(-\chi)$, the origin $\chi=0$ is a singular point where the function and its first derivative must be continuous. This fact suggests to break the infinite range into the left $(\chi \leq 0)$ and right $(\chi \geq 0)$ ranges, having different differential equation for each one. For the left-hand side of the shutter, $\chi \leq 0$, we define $\phi_{<}(\chi, s)$ as the solution of the differential equation:

$$
\frac{\mathrm{d}^{2} \phi_{<}}{\mathrm{d} \chi^{2}}-\left(s^{2}+1\right) \phi_{<}=-(s-i \Omega) \mathrm{e}^{i \kappa \chi},
$$

and for the right-hand side, $\chi \geq 0$, we define $\phi_{>}(\chi, s)$ as the solution of

$$
\frac{\mathrm{d}^{2} \phi_{>}}{\mathrm{d} \chi^{2}}-\left(s^{2}+1\right) \phi_{>}=0 .
$$

Here both functions $\phi_{<}$and $\phi>$ must be bounded: $\left(\phi_{<}\right.$at $\left.\chi \rightarrow-\infty\right)$ and $(\phi$ at $\chi \rightarrow+\infty)$. The important boundary condition is that the two functions and their corresponding first derivatives must be continuous at the interface, $\chi=0$.

Equations (17) and (18) are ordinary second order differential equations and their solution is readily obtained. Taking into account the boundary conditions at $\pm \infty$ we have:

$$
\phi_{<}(\chi, s)=A \mathrm{e}^{\chi \sqrt{s^{2}+1}}+\frac{1}{s+i \Omega} \mathrm{e}^{i \kappa \chi} \quad(\chi \leq 0)
$$

and

$$
\phi_{>}(\chi, s)=B \mathrm{e}^{-\chi \sqrt{s^{2}+1}} \quad(\chi \geq 0)
$$

The constants $A$ and $B$ are fixed from the continuity conditions at the interface $\chi=0$ :

$$
\phi_{<}(0, s)=\phi_{>}(0, s), \quad \mathrm{d} \phi_{<}(0, s) / \mathrm{d} \chi=\mathrm{d} \phi_{>}(0, s) / \mathrm{d} \chi
$$

We have a set of coupled algebraic equations for the constants $A$ and $B$ :

$$
\begin{gathered}
A+\frac{1}{s+i \Omega}=B \\
A \sqrt{s^{2}+1}+\frac{i \kappa}{s+i \Omega}=-B \sqrt{s^{2}+1}
\end{gathered}
$$

with solutions given by:

$$
2 A=\frac{-1}{s+i \Omega}\left(1+\frac{i \kappa}{\sqrt{s^{2}+1}}\right)
$$




$$
2 B=\frac{1}{s+i \Omega}\left(1-\frac{i \kappa}{\sqrt{s^{2}+1}}\right)
$$

Substituting Equation (23) into Equation (19) and Equation (24) into Equation (20) we get, in space $(\chi, s)$, the exact solution for relativistic diffraction in time.

For $(\chi \leq 0)$ we have the solution:

$$
\phi_{<}(\chi, s)=\frac{-1}{2} \frac{1}{s+i \Omega}\left(1+\frac{i \kappa}{\sqrt{s^{2}+1}}\right) \mathrm{e}^{\chi \sqrt{s^{2}+1}}+\frac{1}{s+i \Omega} \mathrm{e}^{i \kappa \chi},
$$

and for $(\chi \geq 0)$ we have:

$$
\phi_{>}(\chi, s)=\frac{1}{2} \frac{1}{s+i \Omega}\left(1-\frac{i \kappa}{\sqrt{s^{2}+1}}\right) \mathrm{e}^{-\chi \sqrt{s^{2}+1}} .
$$

To get the time solution in the variable $\tau$, we must invert the Laplace transforms. We readily find in Laplace Transforms Tables [11] the following results.

$$
\begin{gathered}
\mathcal{L}^{-1}\left[\frac{1}{s+i \Omega}\right]=\mathrm{e}^{-i \Omega \tau} \\
\mathcal{L}^{-1}\left[\mathrm{e}^{-|\chi| \sqrt{s^{2}+1}}\right]=\delta(\tau-|\chi|)-|\chi| \frac{J_{1}\left(\sqrt{\tau^{2}-\chi^{2}}\right)}{\sqrt{\tau^{2}-\chi^{2}}} \theta(\tau-|\chi|) \\
\mathcal{L}^{-1}\left[\frac{\mathrm{e}^{-|\chi| \sqrt{s^{2}+1}}}{\sqrt{s^{2}+1}}\right]=J_{0}\left(\sqrt{\tau^{2}-\chi^{2}}\right) \theta(\tau-|\chi|)
\end{gathered}
$$

where $J_{0}(x)$ and $J_{1}(x)$ denote Bessel functions. Next, using the convolution theorem, and after some simplifications we have:

$$
\mathcal{L}^{-1}\left[\frac{\mathrm{e}^{-|\chi| \sqrt{s^{2}+1}}}{(s+i \Omega) \sqrt{s^{2}+1}}\right]=\theta(\tau-|\chi|) \int_{|\chi|}^{\tau} \mathrm{d} u \mathrm{e}^{-i \Omega(\tau-u)} J_{0}\left(\sqrt{u^{2}-\chi^{2}}\right)
$$

and

$$
\mathcal{L}^{-1}\left[\frac{\mathrm{e}^{-|\chi| \sqrt{s^{2}+1}}}{s+i \Omega}\right]=\theta(\tau-|\chi|)\left[\mathrm{e}^{-i \Omega(\tau-|\chi|)}-|\chi| \int_{|\chi|}^{\tau} \mathrm{d} u \mathrm{e}^{-i \Omega(\tau-u)} \frac{J_{1}\left(\sqrt{u^{2}-\chi^{2}}\right)}{\sqrt{u^{2}-\chi^{2}}}\right]
$$

With the help of Equations (30) and (31) we have the final solutions:

For $\chi \leq 0$ we get, as expected, the incident and reflected wave:

$$
\begin{aligned}
\psi_{<}(\chi, \tau)= & \mathrm{e}^{i(\kappa \chi-\Omega \tau)}-\frac{1}{2} \theta(\tau+\chi)\left[\mathrm{e}^{-i \Omega(\chi+\tau)}+\chi \int_{-\chi}^{\tau} \mathrm{d} u \mathrm{e}^{i \Omega(u-\tau)} \frac{J_{1}\left(\sqrt{u^{2}-\chi^{2}}\right)}{\sqrt{u^{2}-\chi^{2}}}\right. \\
& \left.+i \kappa \int_{-\chi}^{\tau} \mathrm{d} u \mathrm{e}^{i \Omega(u-\tau)} J_{0}\left(\sqrt{u^{2}-\chi^{2}}\right)\right],
\end{aligned}
$$

and for $\chi \geq 0$ we get the transmitted wave: 


$$
\begin{aligned}
\psi_{>}(\chi, \tau)= & \frac{1}{2} \theta(\tau-\chi)\left[\mathrm{e}^{i \Omega(\chi-\tau)}-\chi \int_{\chi}^{\tau} \mathrm{d} u \mathrm{e}^{-i \Omega(\tau-u)} \frac{J_{1}\left(\sqrt{u^{2}-\chi^{2}}\right)}{\sqrt{u^{2}-\chi^{2}}}\right. \\
& \left.-i \kappa \int_{\chi}^{\tau} \mathrm{d} u \mathrm{e}^{-i \Omega(\tau-u)} J_{0}\left(\sqrt{u^{2}-\chi^{2}}\right)\right]
\end{aligned}
$$

We see that in fact, the $1 \mathrm{D}$ shutter problem is nothing but a particular timedependent scattering problem, and here we have the exact relativistic solution.

\section{Conflicts of Interest}

The author declares no conflicts of interest regarding the publication of this paper.

\section{References}

[1] Hegerfeldt, G.C. and Plenio, M.B. (1994) Quantum Optics: Journal of the European Optical Society Part B, 6, 15. https://doi.org/10.1088/0954-8998/6/1/003

[2] Carter, R.E. and Huber, J.R. (2000) Chemical Society Reviews, 29, 305-314. https://doi.org/10.1039/a900724e

[3] Villavicencio, J. and Hernández-Maldonado, A. (1996) Physical Review A, 54, 3055. https://doi.org/10.1103/PhysRevA.101.042109

[4] Moshinsky, M. (1951) Physical Review, 84, 525. https://doi.org/10.1103/PhysRev.84.525

[5] Schneble, D., Hasuo, M., Anker, T., Pfau, T. and Mlynek, J. (2003) Journal of the Optical Society of America B, 20, 648-651. https://doi.org/10.1364/JOSAB.20.000648

[6] Brouard, M. and Muga, J.G. (1996) Physical Review A, 54, 3055. https://doi.org/10.1103/PhysRevA.54.3055

[7] Delgado, F., Cruz, H. and Muga, J.G. (2002) Journal of Physics A: Mathematical and General, 35, 1037. https://doi.org/10.1088/0305-4470/35/48/311

[8] Garca-Calderón, G., Rubio, G. and Villavicencio, J. (1999) Physical Review A, 59, 1758. https://doi.org/10.1103/PhysRevA.59.1758

[9] Delgado, F., Muga, F., Ruschhaput, A., Garca-Calderón, G. and Villavicencio, J. (2003) Physical Review A, 68, Article ID: 032101. https://doi.org/10.1103/PhysRevA.68.032101

[10] Born, M. and Wolf, E. (1965) Principles of Optics. Pergamon Press, Oxford.

[11] Abramowitz, M. and Stegun, I.A. (1965) Handbook of Mathematical Functions. Dover Publications, New York. 